\title{
Excited state dipole moments of anisole in gas phase and solution
}

\author{
Mirko Matthias Lindic ${ }^{\mathrm{a}}$, Matthias Zajonz ${ }^{\mathrm{a}}$, Marie-Luise Hebestreit ${ }^{\mathrm{a}}$, Michael Schneider ${ }^{\mathrm{a}}$, \\ W. Leo Meerts ${ }^{\mathrm{b}}$, Michael Schmitt ${ }^{\mathrm{a}, *}$ \\ a Heinrich-Heine-Universität, Institut für Physikalische Chemie I, D-40225 Düsseldorf, Germany \\ ${ }^{\mathrm{b}}$ Radboud University, Institute for Molecules and Materials, Felix Laboratory, Toernooiveld 7c, 6525 ED Nijmegen, The Netherlands
}

\section{A R T I C L E I N F O}

\section{Keywords:}

Excited state

Dipole moment

Stark spectroscopy

Thermochromic shifts

\begin{abstract}
A B S T R A C T
The excited state dipole moment of anisole has been determined in the gas phase from electronic Stark spectroscopy and in solution using thermochromic shifts in ethyl acetate. Electronic excitation increases the anisole dipole moment in the gas phase from $1.26 \mathrm{D}$ in the ground state to $2.19 \mathrm{D}$ in the electronically excited singlet state, leaving the orientation of the dipole moment practically unchanged. These values are compared to solution phase dipole moments. From variation of the fluorescence emission and absorption maxima with temperature, an excited state dipole moment of $2.7 \mathrm{D}$ was determined. Several solvent polarity functions have been used in combination with experimentally determined cavity volumes at the respective temperatures. Both gas phase and condensed phase experimental dipole moments are compared to the results of $a b$ initio calculations at the CC2 level of theory, using the cc-pVTZ basis set for the isolated molecule and using the COnductor-like Screening MOdel (COSMO), implemented in Turbomole, for the solvated anisole molecule.
\end{abstract}

\section{Introduction}

The use of dipole moments as measures for the distribution of electrons in molecules has successfully be applied for more than 100 years. The earliest designation of the concept of molecular dipoles goes back to Debye's paper of 1912 on a kinetic theory of insulators [1]. However, this concept is not uncontroversial, since for larger molecules higher terms of the multipole expansion may be needed to properly describe molecular electronic properties. The success of the model can be traced back to the fact, that the overall dipole moment can be constructed from atomic dipoles, which are centered at the atomic positions and point along the chemical bonds, the so called bond dipoles [2]. The main problem concerning the molecular dipole moments, however, results from the fact, that their experimental determination should preferably be performed in the vapor phase to avoid interaction with the surrounding solvent. Many substances of interest decompose thermally, before their vapor pressure is high enough to allow for the experimental determination of the dipole. In these cases determination of the molecular dipole in dilute solutions is the only possibility. For the ground state several schemes exist, to obtain vapor-phase dipole moments from solution measurements [3]. They can be applied to strongly dilute solutions with nonpolar solvents. For the excited state, the situation is much more complex, since mutual solvent-solute interactions are a prerequisite in many techniques used for the determination of excited state dipole moments in solution [4].

The knowledge of excited state dipole moments of molecules and of transition dipole moments connecting ground and excited states are important prerequisites for the understanding of resonance energy transfer processes like Förster Resonant Energy Transfer (FRET) [5,6] and for molecular excitonic interactions [7]. While determination of permanent dipole moments in the electronic ground state of molecules is straightforward using microwave spectroscopy, exact dipole moments in electronically excited states are much more difficult to be determined. The probably most exact method is rotationally resolved electronic Stark spectroscopy [8-12]. This method has the advantage to yield both the ground and excited state dipole moments with an accuracy of about $1 \%$. The applied field strengths in these experiments are - depending on the size of the dipole moment - in the range of $100-1000 \mathrm{~V} / \mathrm{cm}$, what allows to treat the electric field induced changes to the rotational Hamiltonian as perturbations. However, this method can only be applied to isolated molecules in the gas phase at considerably low temperatures as obtained in molecular beams.

Historically, excited-state dipole moments in solution have been determined from solvatochromic shifts in solvents of different dielectric constants $[13,14]$ or from electro-optical absorption measurements $[15,16]$. The evaluation of excited state dipole moments from solvatochromic shifts applies the so called Lippert-Mataga equation [17,18]. The derivation of this equation is based on Onsager's reaction field [19],

\footnotetext{
* Corresponding author.

E-mail address: mschmitt@hhu.de (M. Schmitt).
} 
which assumes the fluorophore to be a point dipole, which is located in the center of a spherical cavity. The cavity of radius $a$ is formed by the homogeneous and isotropic solvent with the permittivity $\epsilon$. The molecular dipole moment of the solute induces a dipole in the homogeneous solvent. This (reaction) dipole is the source of an electric field, which interacts with the molecular dipole, leading to an energetic stabilization. In the case of anisole, the cavity volume is about $1.79 \times 10^{-28} \mathrm{~m}^{3}$ equivalent to a cavity radius of $4.2 \times 10^{-10} \mathrm{~m}$. With a reaction dipole of $1.3 \mathrm{D}$, a field strength in the cavity of $5 \times 10^{8} \mathrm{~V} / \mathrm{m}$ is reached. This field strength is more than 1000 times higher than the fields used in gas phase Stark spectroscopy.

This model already defines the limits of the method, since the original Lippert-Mataga theory does not account for non-spherical cavities, expanded dipoles in the cavity, specific interactions between the solute and the solvent like hydrogen bonds, etc., and reorientation of the molecular dipole upon electronic excitation. Numerous improvements to the basic Lippert-Mataga theory have been introduced over the years, like the utilization of multi-parameter solvent polarity scales, which quantitatively reproduce solvent-solute interactions. These interactions are modulated through linear solvation energy relationships $[20,21]$. An improved model by Abe also allows for a determination of the angle $\theta$ between the ground and excited state dipole moments [22]. All improvements add several new parameters, which are mainly empirical, like scaling factors for the solvent hydrogen solvent bond donor acidities and basicities, empirical polarity parameters of the solvents, and the volume variation of cavity with solvent polarity to account for non-spherical cavities [23]. Apart from improvements of the solvent functions used, Kawski introduced the method of thermochromic shifts in spite of solvatochromic shifts, in order to minimize the effect of different solvents on the Onsager radius [24].

The structures of the anisole chromophore in the ground and lowest excited singlet states have been studied by high resolution spectroscopy before using rotationally resolved fluorescence excitation spectroscopy in the groups of Becucci [25] and Pratt [26]. Also, triply deuterated anisole (deuterated at the methoxy group $\mathrm{OCD}_{3}$ ) was studied in high resolution by Pasquini et al. [27]. However, exact dipole data of anisole are available only for the ground state from a microwave Stark experiment [28].

In the present study, excited state dipole moments of anisole from electronic Stark spectroscopy in the gas phase are compared to those measured in solution using thermochromic shifts. Anisole was chosen as test molecule, because it combines several advantages for this comparison. First, it is small enough to facilitate evaporation and gas phase spectroscopic investigations. Secondly, the angle of the dipole moment of anisole in the molecular frame stays nearly constants upon electronic excitation. Third, it dissolves well in aprotic solvents, to minimize effects due to hydrogen bond formation between solute and solvent. The final goal is to extend and calibrate methods for the determination of dipole moments of large molecules in solution to the limit in which they cannot be determined anymore by means of gas phase spectroscopy.

\section{Computational methods}

\subsection{Quantum chemical calculations}

Structure optimizations were performed employing Dunning's correlation consistent polarized valence triple zeta (cc-pVTZ) basis set from the tuRBomole library $[29,30]$. The equilibrium geometries of the electronic ground and the lowest excited singlet states were optimized using the second order approximate coupled cluster model (CC2) employing the resolution-of-the-identity (RI) approximation [31-33]. Dipole moments are calculated as first derivatives of the respective energy with respect to an external field at the CC2 level of theory. The Conductor-like Screening Model (COSMO) [34], which is implemented in the ricc 2 module of the tURBOmole package, was used for the calculation of ground and excited state dipole moments in solution.

\subsection{Fits of the rovibronic spectra using evolutionary algorithms}

Evolutionary algorithms have proven to be perfect tools for the automated fit of rotationally resolved spectra, even for large molecules and dense spectra [35-38]. Beside a correct Hamiltonian to describe the spectrum and reliable intensities inside the spectrum, an appropriate search method is needed. Evolutionary strategies are powerful tools to handle complex multi-parameter optimizations and find the global optimum. For the analysis of the presented high-resolution spectra we used the covariance matrix adaptation evolution strategy (CMA-ES), which is described in detail elsewhere $[39,40]$. In this variant of global optimizers mutations are adapted via a covariance matrix adaptation (CMA) mechanism to find the global minimum even on rugged search landscapes that are additionally complicated due to noise, local minima and/or sharp bends. The analysis of the rotationally resolved electronic Stark spectra is described in detail in Ref. [41]. The fit of the rovibronic Stark spectra with $\Delta M=0$ and $\Delta M= \pm 1$ selection rules can be fit simultaneously, improving both the accuracy and reducing the correlation between the fitted parameters. Additionally, we implemented the use of boundary conditions in the simultaneous fits of Stark spectra of different isotopologues. Since the absolute values of the dipole moments $|\mu|$ in the ground and excited state depend only weakly on the different isotopologues, the parameters $\left|\mu^{g}\right|$ and $\left|\mu^{e}\right|^{1}$ can be set equal leaving only the components $\left|\mu_{a}^{g, e}\right|$ (isotopologue 1), $\left|\mu_{a}^{g, e}\right|$ (isotopologue 2), $\left|\mu_{b}^{g, e}\right|$ (isotopologue 1) and $\left|\mu_{b}^{g, e}\right|$ (isotopologue 2) to be fit.

\subsection{Evaluation of excited state dipole moments from thermochromic shifts}

The limitations utilizing solvatochromic shifts for determination of excited state dipole moments have been discussed thoroughly $[17,18,42,22,43,24,23]$. To circumvent the problems of non-constant cavity sizes when using different solvents, Kawski [24] proposed to use the influence of the temperature on the permittivity and index of refraction of the solvent to induce the spectral shifts of the solutions. Still, the problem remains, that small variations of the Onsager radius result in large errors of the excited state dipole moments, since the Cavity radius enters the Lippert-Mataga [17,18] and the Bilot-Kawski [43,24] equations in the third power. Therefore, Demissie [23] proposed using the real cavity volume instead of a spherical cavity calculated from Onsager radius [19]. The real cavity volume is determined experimentally from concentration dependent density measurements which are evaluated using Eq. (1).

$\frac{1}{\rho}=\frac{1}{\rho^{*}}+\left(\frac{V_{m}}{M}-\frac{1}{\rho^{*}}\right) \cdot w$

$\rho$ is the measured density of the solution, $\rho^{*}$ is the density of the pure solvent and $w$ is the mass fraction of the solvate. The slope of the graph $\frac{1}{1}(w)$ gives the cavity volume per mole $V_{m}$ of the solvate. This procedure has been applied at all temperatures, for which spectral shift data were obtained.

The original equation for solvatochromic shifts according to Lippert-Mataga can be used for thermochromic shifts as well, when the temperature dependence of $\epsilon$ and $n$ is known:

$\widetilde{\nu}_{A}-\widetilde{\nu}_{F}=-\frac{2\left(\mu_{e}-\mu_{g}\right)^{2}}{4 \pi \varepsilon_{0} \mathrm{~h} \mathrm{c} a^{3}} \cdot F_{\mathrm{LM}}+$ const.

where $\varepsilon_{0}$ is the vacuum permittivity, $h$ the Planck constant, $c$ the lights speed, $a$ the Onsager cavity radius, and $F_{L M}$ the solvent polarity function according to Lippert and Mataga [17,18]:

$F_{\mathrm{LM}}=\frac{\varepsilon-1}{2 \varepsilon+1}-\frac{1}{2}\left(\frac{n^{2}-1}{2 n^{2}+1}\right)$

\footnotetext{
${ }^{1}$ The superscript " $\mathrm{g}$ " and "e" refer to the electronic ground and excited state, respectively.
} 
The modified equation from Kawski [24] has also been used:

$\widetilde{\nu}_{A}+\widetilde{\nu}_{F}=-\frac{2\left(\mu_{e}^{2}-\mu_{g}^{2}\right)}{4 \pi \varepsilon_{0} \mathrm{~h} \mathrm{c} a^{3}} \cdot F_{\mathrm{BK}}+$ const.

where $F_{B K}$ the solvent polarity function according to Bilot and Kawski $F_{B K}[43]$ :

$F_{\mathrm{BK}}=\left[\frac{\varepsilon-1}{\varepsilon+2}-\frac{n^{2}-1}{n^{2}+2}\right] \frac{2 n^{2}+1}{2\left(n^{2}+2\right)}+\frac{3}{2}\left[\frac{\left(n^{4}-1\right)}{\left(n^{2}+2\right)^{2}}\right]$

with $\varepsilon$ as solvent permittivity and $n$ as refractive index of the solvent.

Using the cavity volume per mole $V_{m}$, Eqs. (4) and (5) can be rearranged to include the single molecule cavity volume $V=4$ / $3 \pi a^{3}=V_{m} / N_{A}$ into the solvent polarity function. Eq. (4) is then modified to:

$\widetilde{\nu}_{A}+\widetilde{\nu}_{F}=-\frac{2\left(\mu_{e}^{2}-\mu_{g}^{2}\right)}{3 \varepsilon_{0} \mathrm{~h} \mathrm{c}} \cdot F_{\mathrm{BKD}}+$ const.

and the solvent polarity function (5) to:

$F_{\mathrm{BKD}}=\frac{1}{V}\left(\frac{2 n^{2}+1}{n^{2}+2}\left(\frac{\varepsilon-1}{\varepsilon+1}-\frac{n^{2}-1}{n^{2}+2}\right)+\frac{3\left(n^{4}-1\right)}{\left(n^{2}+2\right)^{2}}\right)$

The modified thermochromic equation, which will be used in the evaluation of the dipole moments according to Demissie [23] is:

$\tilde{\nu}_{A / E}(T)=\widetilde{\nu}_{A / E}^{0}(T)-\frac{2 \mu_{g / e}\left(\mu_{e}-\mu_{g}\right)}{3 \varepsilon_{0} \mathrm{hc}} \cdot F_{\mathrm{BKD}}(T)$

with

$F_{\mathrm{BKD}}(T)=\frac{1}{V}\left(\frac{2 n(T)^{2}+1}{n(T)^{2}+2}\left(\frac{\varepsilon(T)-1}{\varepsilon(T)+1}-\frac{n(T)^{2}-1}{n(T)^{2}+2}\right)+\frac{3\left(n(T)^{4}-1\right)}{\left(n(T)^{2}+2\right)^{2}}\right)$

The temperature dependent values of $\varepsilon$ and $n$ were determined by Gryczyński and Kawski [44]. The slope $m$ of $\left(\widetilde{\nu}_{A}+\widetilde{\nu}_{F}\right)$ versus $F_{B K}$ using the experimentally determined ground state dipole moment now gives the excited state dipole moment (from Eqs. (4) and (5)):

$\mu_{e}=\sqrt{\mu_{g}^{2}+\frac{3 \varepsilon_{0} \cdot h \cdot c \cdot m}{2}}$

Using Eqs. (8) and (6), the slopes $m_{A}$ and $m_{F}$ of the respective plots of $\widetilde{\nu}_{A}(T)$ and $\widetilde{\nu}_{E}(T)$ vs. the solvent polarity functions $F_{B K}(T)$ or $F_{B K D}(T)$ yield the dipole moments of ground and excited state independently:

$\mu_{g}=\sqrt{\frac{3 \varepsilon_{0} \mathrm{hcm}_{A}^{2}}{2\left(m_{F}-m_{A}\right)}}$

$\mu_{e}=\sqrt{\frac{3 \varepsilon_{0} \mathrm{hcm}_{F}^{2}}{2\left(m_{F}-m_{A}\right)}}$

\section{Experimental methods}

\subsection{Electronic Stark spectroscopy}

Anisole ( $\geq 99 \%$ ) was purchased from Carl Roth $\mathrm{GmbH}$ and used without further purification. $d_{3}$-anisole $(99.3 \%)$ was purchased from $\mathrm{CDN}$ isotopes. The sample was heated to $313 \mathrm{~K}$ and co-expanded with 200-300 mbar of argon into the vacuum through a $200 \mu \mathrm{m}$ nozzle. After the expansion a molecular beam was formed using two skimmers $(1 \mathrm{~mm}$ and $3 \mathrm{~mm}, 330 \mathrm{~mm}$ apart) linearly aligned inside a differentially pumped vacuum system consisting of three vacuum chambers. The molecular beam was crossed at right angles with the laser beam $360 \mathrm{~mm}$ downstream of the nozzle. To create the excitation beam, $10 \mathrm{~W}$ of the $532 \mathrm{~nm}$ line of a diode pumped solid state laser (Spectra-Physics Millennia eV) pumped a single frequency ring dye laser (Sirah Matisse DS) operated with Rhodamine 110. The light of the dye laser was frequency doubled in an external folded ring cavity (Spectra Physics Wavetrain) with a resulting power of about $25 \mathrm{~mW}$. The fluorescence of the samples was collected perpendicular to the plane defined by laser and molecular beam using an imaging optics setup consisting of a concave mirror and two plano-convex lenses onto the photocathode of a UV enhanced photomultiplier tube (Thorn EMI 9863QB). The signal output was then discriminated and digitized by a photon counter and transmitted to a PC for data recording and processing. The relative frequency was determined with a quasi confocal Fabry-Perot interferometer. The absolute frequency was obtained by comparing the recorded spectrum to the tabulated lines in the iodine absorption spectrum [45]. A detailed description of the experimental setup for the rotationally resolved laser induced fluorescence spectroscopy has been given previously $[46,47]$.

To record rotationally resolved electronic Stark spectra, a parallel pair of electro-formed nickel wire grids (18 mesh per mm, $50 \mathrm{~mm}$ diameter) with a transmission of $95 \%$ in the UV was placed inside the detection volume, one above and one below the molecular beam - laser beam crossing with an effective distance of $23.49 \pm 0.05 \mathrm{~mm}$ [41]. In this setup the electric field is parallel to the polarization of the laser radiation. With an achromatic $\lambda / 2$ plate (Bernhard Halle, $240-380 \mathrm{~nm}$ ), mounted on a linear motion vacuum feedthrough, the polarization of the incoming laser beam can be rotated by $90^{\circ}$ inside the vacuum.

\subsection{Thermochromic shifts}

The density $\rho$ of the solution of anisole in ethyl acetate was measured at different mass fractions $w$ in a temperature range of $263 \mathrm{~K}$ and $343 \mathrm{~K}$ with $2 \mathrm{~K}$ increment using a density meter (Anton Paar DMA4500M). The cavity volume is determined from the slope of the graph of $\frac{1}{\rho}$ vs. the mole fraction $w$ at each temperature. A plot of the inverse density of the solution of anisole in ethyl acetate versus the weight fraction of anisole along with the linear fit of the data is shown in Fig. S1 of the Ref. [48].

Absorption spectra of anisole, dissolved in ethyl acetate have been recorded in a temperature range of $258 \mathrm{~K}$ and $348 \mathrm{~K}$ with $5 \mathrm{~K}$ increment using a Varian Cary 50 Scan UV spectrometer. A custom built coolable and heatable cell holder has been constructed using a pair of Peltier elements from Uwe Electronics GmbH. The hot side was cooled using a Julabo Corio 600F cooler with Thermal G as cooling liquid. The cell holder is mounted in a vacuum chamber to avoid condensation of water on the windows at low temperatures. Fluorescence emission spectra were recorded in a Varian Cary Eclipse spectrometer using the same cell holder as for the absorption measurements.

\section{Results}

\subsection{Computational results}

The structures of anisole in the electronic ground and excited states have been optimized at the CC2/cc-pVTZ level of theory. The Cartesian coordinates of all optimized structures are given in Tables S5 and S6 of the Ref. [48]. Table 1 summarizes the results on structures and dipole moments of anisole along with the experimental results. The rotational constants as measures of the quality of the CC2/cc-pVTZ optimized structures in the ground and first electronically excited state agree well with the experimentally determined ones, cf. Table 1 . The absolute permanent dipole moment of the isolated anisole molecule in the electronic ground state was calculated to $1.33 \mathrm{D}$. The projections of the dipole moment onto the inertial $a$ and $b$-axes (cf. Fig. 1) are calculated to be $0.64 \mathrm{D}$ and $1.17 \mathrm{D}$, respectively.

From these projections an angle $\theta_{D}^{\prime}$ of $+61^{\circ}$ of the dipole moment with the inertial $a$-axis is obtained in the electronic ground state. A positive sign of this angle means an anticlockwise rotation of the dipole moment vector onto the main inertial $a$-axis for the molecular 
Table 1

Calculated rotational constants, permanent electric dipole moments $\mu$ and their components $\mu_{i}$ along the main inertial axes $i=a, b, c$ of anisole compared to the respective experimental values. Doubly primed parameters belong to the electronic ground and single primed to the excited state. $\theta_{D}$ is the angle of the dipole moment vector with the main inertial $a$-axis. A positive sign of this angle means a clockwise rotation of the dipole moment vector onto the main inertial $a$-axis.

\begin{tabular}{|c|c|c|c|c|c|c|c|c|c|}
\hline & \multicolumn{3}{|l|}{ Theory } & \multicolumn{6}{|l|}{ Experiment } \\
\hline & \multicolumn{2}{|l|}{ Isolated } & \multirow{2}{*}{$\begin{array}{l}\text { COSMO } \\
h_{3}\end{array}$} & \multicolumn{2}{|l|}{ Gas phase } & \multicolumn{4}{|c|}{ Thermochromic shifts $^{\mathrm{d}}$} \\
\hline & $h_{3}$ & $d_{3}$ & & $h_{3}$ & $d_{3}$ & {$[1]$} & {$[2]$} & [3] & [4] \\
\hline$A^{\prime \prime} / \mathrm{MHz}$ & 5023 & 4846 & - & $5028.84414(19)^{\mathrm{a}}$ & $4850.68(8)$ & - & - & - & - \\
\hline$B^{\prime \prime} / \mathrm{MHz}$ & 1579 & 1448 & - & $1569.364308(68)^{\mathrm{a}}$ & $1438.81(2)$ & - & - & - & - \\
\hline$C^{\prime} / \mathrm{MHz}$ & 1211 & 1131 & - & $1205.825614(41)^{\mathrm{a}}$ & $1126.03(2)$ & - & - & - & - \\
\hline$\mu_{a}^{\prime \prime} / \mathrm{D}$ & +0.64 & +0.66 & - & $\pm 0.6937^{\mathrm{a}}$ & $\pm 0.79(6)$ & - & - & - & - \\
\hline$\mu_{b}^{\prime \prime} / \mathrm{D}$ & +1.17 & +1.15 & - & $\pm 1.0547^{\mathrm{a}}$ & $\pm 0.98(3)$ & - & - & - & - \\
\hline$\mu^{\prime \prime} / \mathrm{D}$ & 1.33 & 1.33 & 1.12 & $1.2623^{\mathrm{a}}$ & 1.2623 & $1.17^{\mathrm{c}}$ & $1.17^{\mathrm{c}}$ & $0.19(3)$ & $0.40(5)$ \\
\hline$\theta_{D}^{\prime \prime} /^{\circ}$ & +61 & +60 & - & \pm 56.7 & \pm 51.3 & - & - & - & - \\
\hline$A^{\prime} / \mathrm{MHz}$ & 4777 & 4621 & - & 4795.17(13) & $4636.82(11)$ & - & - & - & - \\
\hline$B^{\prime} / \mathrm{MHz}$ & 1567 & 1436 & - & $1555.68(4)$ & $1310.97(3)$ & - & - & - & - \\
\hline $\mathrm{C}^{\prime} / \mathrm{MHz}$ & 1189 & 1111 & - & $1184.45(3)$ & $934.39(3)$ & - & - & - & - \\
\hline$\mu_{a}^{\prime} / \mathrm{D}$ & +1.20 & +1.23 & - & $\pm 1.59(3)$ & $\pm 1.76(1)$ & - & - & - & - \\
\hline$\mu_{b}^{\prime} / \mathrm{D}$ & +1.95 & +1.94 & - & $\pm 1.50(3)$ & $\pm 1.30(2)$ & - & - & - & - \\
\hline$\mu^{\prime} / \mathrm{D}$ & 2.30 & 2.30 & 2.01 & $2.19(4)$ & 2.19 & $2.7(2)$ & $6.6(3)$ & $2.4(2)$ & $4.9(3)$ \\
\hline$\theta_{D}^{\prime} /^{\circ}$ & +58 & +57 & - & \pm 43.4 & \pm 36.5 & - & - & - & - \\
\hline$\nu_{0} / \mathrm{cm}^{-1}$ & $37,179^{b}$ & $37,184^{b}$ & - & $36,384.07(1)$ & $36,387.31(1)$ & - & - & - & - \\
\hline
\end{tabular}

a Set to the microwave values from Ref. [28].

b Adiabatic excitation energy, including ZPE corrections.

c From Ref. [49].

${ }^{\mathrm{d}}$ [1] = Kawski; [2] = Lippert-Mataga; [3] = Demissie with Bilot-Kawski polarity function; [4] = Demissie with Lippert-Mataga polarity function. For details see text.



Fig. 1. Structure, inertial axis system and dipole moment in ground (red, straight) and first electronically excited singlet state (blue, dotted) of anisole. The dipole vector is drawn from negative to positive. The primed coordinate system (black, dashed) refers to the $d_{3}$ isotopologue. The positive direction of the dipole moment angle $\theta_{D}^{\prime}$ in the ground state and $\theta_{D}^{\prime}$ in the excited state are shown. (For interpretation of the references to color in this figure legend, the reader is referred to the web version of this article.)

orientation, shown in Fig. 1. For the lowest electronically excited singlet state of anisole, dipole moment components of 1.20 and 1.95 D with respect to the inertial $a$ and $b$-axes are calculated, corresponding to an angle $\theta_{D}^{\prime}$ of $+58^{\circ}$. Thus, the angle of the dipole moment within the molecular frame stays nearly constant upon electronic excitation.

Table 1 also reports the results for the triply deuterated $d_{3}$-anisole. In first order, the dipole moment of the deuterated compound will be the same as of the undeuterated one. However, due to the larger mass of the $-\mathrm{CD}_{3}$ group, the inertial $a$ axis is rotated towards the $-\mathrm{CD}_{3}$ group. This opens the possibility to determine the absolute orientation of the dipole moment experimentally. Dipole moment components from Stark measurements have an indeterminacy of the sign of the components, since only the projections onto the inertial axes are obtained. Tilting the axis via isotopic substitution removes the indeterminacy partially, since for both signs of $\mu_{a}$ and $\mu_{b}$ positive, the angle between the dipole moment vector and the $a$ axis decreases, while for different signs of $\mu_{a}$ and $\mu_{b}$ it increases. Thus, the dipole moment points from the origin to the first or third quadrant, if the angle gets smaller upon deuteration.

\subsection{Experimental results}

\subsubsection{Permanent dipole moments of anisole from Stark spectra}

The rotationally resolved electronic spectrum of anisole has been presented and analyzed before in the groups of Becucci and Pratt $[25,26]$. However, no Stark measurements have been performed until now, and the dipole moment in the excited singlet state of the isolated molecule is not known experimentally. We measured and analyzed the electronic Stark spectrum of anisole shown in Fig. 2.

The dipole moments have been obtained from a combined fit to the Stark spectra in parallel as well as in perpendicular arrangement of laser polarization and electric field [41]. The fit using the CMA-ES algorithm yielded the parameters given in Table 1 . The ground state dipole components, which have been obtained by Desyatnyk et al. [28] using microwave Stark spectroscopy, have been kept fixed in our fit, due to the inherently larger accuracy of the MW values. Experimental rotational constants and dipole moments of both electronic states are in good agreement with the results for the CC2//cc-pVTZ optimized structure. From the ratio of intensities of the $a$ and the $b$ lines of anisole, the angle of the excited state dipole moment with the inertial $a$ axis can be determined to be $56.7^{\circ}$ in reasonable agreement with the theoretical value from CC2/cc-pVTZ calculations of $61^{\circ}$. The results of the fits are compiled in Table 1. 




Fig. 2. Rotationally resolved electronic Stark spectrum of the electronic origin of anisole at $36,384.07 \mathrm{~cm}^{-1}$. The upper trace shows the experimental spectrum at zero-field. The second trace shows a zoomed in portion of the spectrum along with the simulated spectrum, using the molecular parameters from Table 1 . The lowest trace contains the Stark spectrum at a field strength of $400.24 \mathrm{~V} / \mathrm{cm}$ with electric field direction and electromagnetic field parallel to each other, hence with $\Delta M=0$ selection rules.

\subsubsection{Electronic Stark spectra of $d_{3}$-anisole}

Electronic Stark spectroscopy yields the projections of the dipole moments in both electronic states onto the inertial axes. No information however, is obtained regarding the sign of the dipole components. The calculated permanent dipole moment of anisole in the electronic ground state, shown in Fig. 1 has both dipole components $\left(\mu_{a}\right.$ and $\left.\mu_{b}\right)$ positive. i.e. the dipole moment vector points to the first quadrant in the chosen orientation of Fig. 1. The sign however, can be obtained from the evaluation of Stark spectra of isotopically substituted anisole. We chose the methoxy-deuterated isotopologue, since the effect of hydrogen-deuterium exchange gives the largest possible isotopic ratio and the effect on the inertial axes of the parent molecule will be large enough to be measured reliably. The $d_{3}$-anisole spectrum has been fit together with the $h_{3}$-anisole under the constraint of equal absolute values of the dipole moment $|\mu|$ in both states. This assumption seems to be justified, since in medium sized molecules dipole moment changes of $0.001-0.01 \mathrm{D}$ are found upon hydrogen-deuterium exchange are reported [50]. The so determined dipole moment components $\mu_{a}$ and $\mu_{b}$ in the ground state are equivalent to an angle of the dipole moment with the inertial $a$-axis of $51.3^{\circ}$. This decrease compared to the value of the $h_{3}$ isotopologue matches the predicted change from the $a b$ initio calculations. Inspection of Fig. 1 shows, that the inertial axes will be rotated towards the dipole moment vector upon $\mathrm{H} \leftrightarrow \mathrm{D}$ interchange, leading to the observed decrease of the angle $\theta_{D}$. The experimentally determined decrease is considerably larger, than the predicted ones, using the CC2 optimized structures, what is probably due to the limitations of the above model.

\subsubsection{Permanent dipole moments of anisole from thermochromic shifts}

In a first step, we determined the cavity volume of anisole in ethyl acetate from equation (1). Fig. S1 of Ref. [48] shows the plot of the inverse density of the solution of anisole in ethyl acetate versus the weight fraction of the solute at $293 \mathrm{~K}$. From the slope of the graph, a cavity volume of $1.79 \times 10^{-28} \mathrm{~m}^{3}$ at $293 \mathrm{~K}$ could be determined. Subsequently, the cavity volume was determined at all temperatures, for which absorption and emission spectra have been taken. The plot of the molar volumes vs. the temperature is shown in Fig. 3.

The absorption and fluorescence emission spectra of anisole,

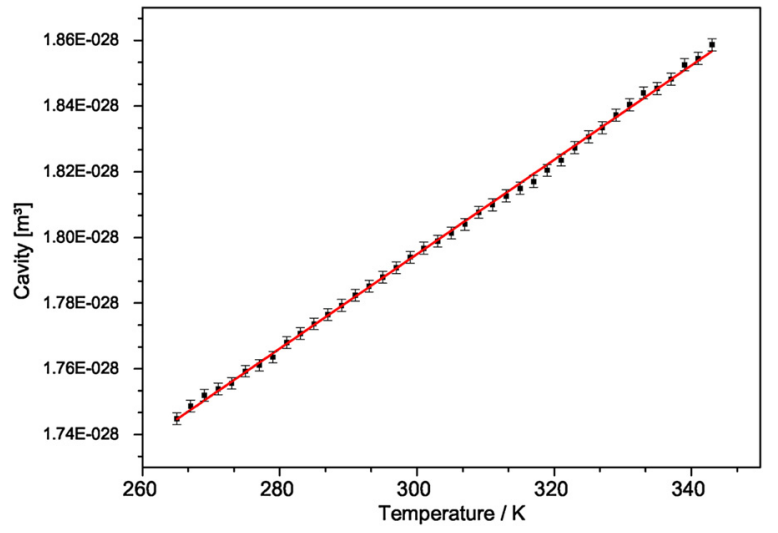

Fig. 3. Plot of the cavity volumes versus temperature of anisole in ethyl acetate along with the linear fit of the data.

dissolved in ethyl acetate at temperatures between $258 \mathrm{~K}$ and $348 \mathrm{~K}$ are shown in Fig. 4. For the evaluation of the maxima of the absorption spectra, they were fit with a set of multiple Gauss functions. We used the lowest energy local maximum of the absorption spectra as $\widetilde{\nu}_{A}$ and the overall maximum of the emission spectra as $\widetilde{\nu}_{F}$ (see Fig. 4).

Several approaches to the changes of the dipole moments upon electronic excitation have been tested. Following equation (4), the sum of wavenumbers $\widetilde{\nu}_{A}+\widetilde{\nu}_{F}$ is plotted versus calculated solvent polarity function $F_{B K D}(T)$, as shown in Fig. 5. The excited state dipole moment can be calculated from its slope $m$ of this plot, using the known ground state dipole moment as shown in equation (10). Using the experimentally determined ground state dipole moment of $1.17 \mathrm{D}$, the excited state dipole moment is determined to be 2.7(2) D using the experimental molar cavity volume and the solvent polarity function of Bilot and Kawski (equation (7)) ([1] in Table 1).

Application of the original Lippert-Mataga plot ([2] in Table 1), an excited state dipole moment of 6.6(3) D is obtained. The modified thermochromic equation according to Demissie with the Bilot-Kawski solvent polarity function ([3] in Table 1) allows for an independent determination of the ground and excited state dipole moments. It yields 


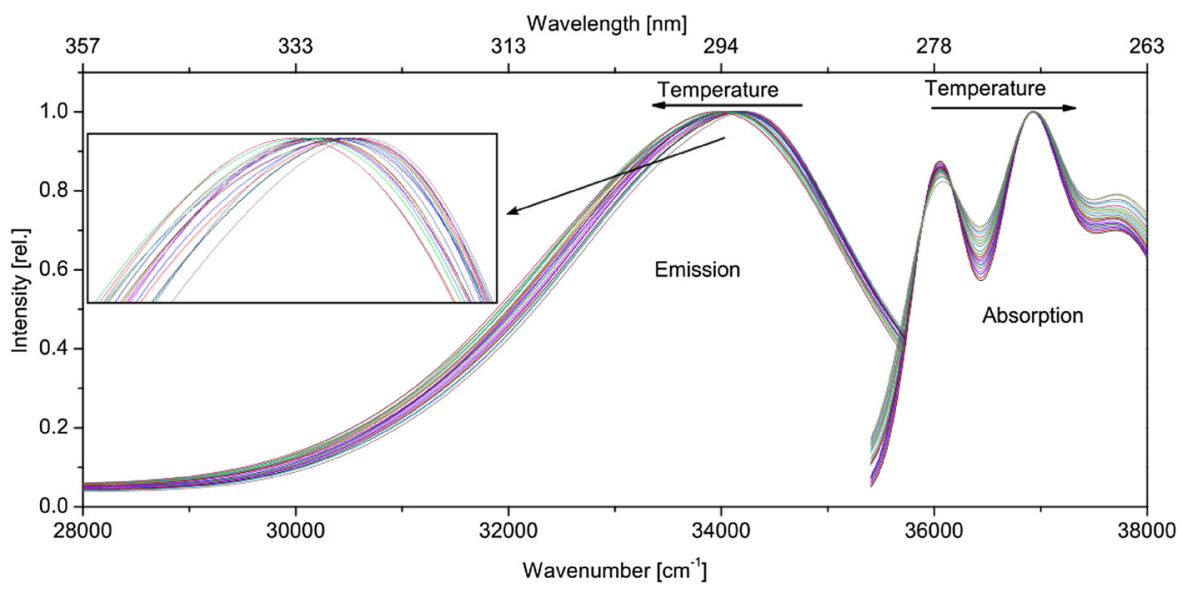

Fig. 4. Absorption and emission spectra of anisole in ethyl acetate between $258 \mathrm{~K}$ and $348 \mathrm{~K}$. The inset shows an enlarged portion of the emission spectra.

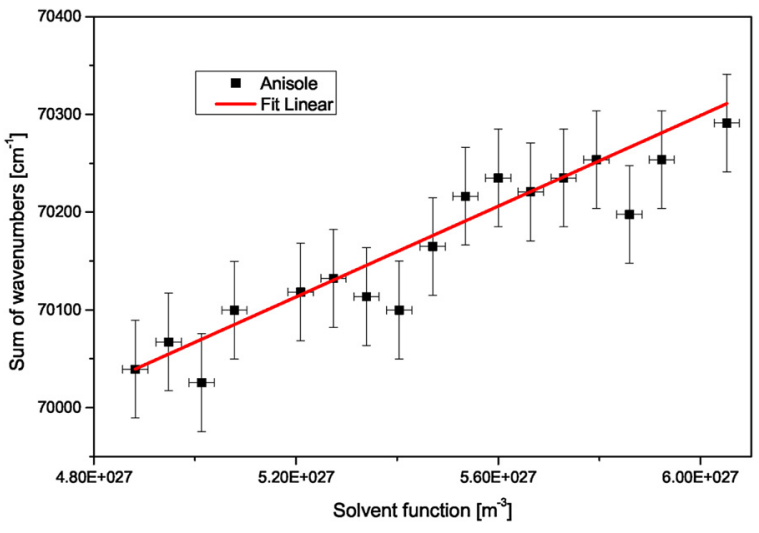

Fig. 5. Plot of $\widetilde{\nu}_{A}+\widetilde{\nu}_{F}$ versus calculated polarity function $F_{B K}$.

$0.19(3) \mathrm{D}$ for the ground state and 2.4(2) D for the electronically excited state. The same equation, but with the Lippert-Mataga solvent polarity function ([4] in Table 1) values of 0.40(4) D and 4.9(3) D for ground and excited state, respectively are obtained.

\section{Discussion}

The excited state dipole moment of anisole has been determined from Stark spectroscopy of anisole and its triply (methyl group) deuterated isotopologue. The absolute value $2.30 \mathrm{D}$ shows that the dipole moment increases by more than $1 \mathrm{D}$ upon electronic excitation. The direction of the dipole changes only slightly upon electronic excitation as can be seen from the experimentally determined dipole components.

The comparison of the experimentally determined excited state dipole moments from electronic Stark measurements and from thermochromic shifts shows best agreement between the gas and condensed phase experimental values using the equations from Demissie's equation along with the Bilot and Kawski solvent function (model [3] in Table 1). However, the ground state dipole moment in solution, which is determined independently using these equations is completely wrong, when compared to the gas phase value from MW spectroscopy. The original Lippert-Mataga model fails completely in describing the excited state dipole moment correctly (model [2] in Table 1). The reason for this large discrepancy concerning the original Lippert-Mataga equation is described nicely by Kawski [51]. For a polarizability of the solute $\alpha=0$ the solvent polarity function of Bilot and Kawski (Ref. $[24,43])$ passes over to the original Lippert-Mataga equation. It can thus safely be stated that it is the neglect of solvent polarizability in Lippert and Mataga's equation, which causes the deviations to the experiment.
Good agreement for the excited state is also obtained using Kawski's equations with the Bilot and Kawski solvent function (model [1] in Table 1). This method yields only changes of the dipole moment upon electronic excitation. The ground state dipole from solution measurements of Ref. [49] has been used.

The last test was performed in order to check, if the Lippert-Mataga solvent function gives reliable results using Demissie's modified equations (model [4] in Table 1). Clearly, in this case ground and excited state dipole moments deviate strongly from the gas phase values.

The cavity volume, which is calculated from the COSMO model, implemented in the ricc 2 module of turbomole is determined to be $1.42 \times 10^{-28} \mathrm{~m}^{3}$ while the experimental value from the measurement of the solution density as function of the weight fraction of anisole is $1.79 \times 10^{-28} \mathrm{~m}^{3}$. The deviation of $21 \%$ might seem small, but since the cavity radius enters in the third power in the original Lippert-Mataga plots, its effect on the excited dipole moment is crucial.

Overall, the Bilot-Kawski method [24,43], modified by Demissie [23] using real cavity volumes is the most promising method for determination of excited state dipole moments in solution. Furthermore it must be emphasized that this method yields through the extrapolation technique of the linear fit to the experimental data values, which correspond to the gas phase. This dipole moment, free from solute-solvent interactions, is the only which can be compared to the gas phase data and to $a b$ initio calculations of the isolated molecule [52]. This work will be extended to molecules, which have a larger difference of the orientation of the dipole moments in ground and excited state. Since most molecules of interest will have arbitrary orientations, the extension of the Bilot-Kawski method in order to include the change of the angle of the dipole moment upon electronic excitation, like in the model of Abe [22].

\section{Conclusions}

The exact determination of dipole moments of molecules in their excited states from rotationally resolved Stark experiments can only be performed with small and/or volatile molecules. Conceptionally and experimentally straightforward approaches like solvatochromic shifts in different solvents with a large variety of different solvent polarity functions have been used in numerous investigations over many decades. However, large deviations between vapor and condensed phase dipole moments have been found in the few thorough comparisons of molecules for which exact gas phase data are available $[53,54]$.

Several reasons for the discrepancy between excited state dipole moments from Stark experiments in the gas phase and solvatochromic shifts have been identified. (i) Many theories, based on the original Onsager and Lippert-Mataga equations use the Onsager radius as free parameter. This radius is neither well known nor well defined, but 
enters the respective equations in cubic power, causing a large error. (ii) The inherently low resolution in solution makes the determination of spectral shifts cumbersome. (iii) Changing the solvent causes inevitably also changes in the interaction between solvent and solute and results in changes of the cavity radius of cavity volume. (iv) The large field strengths in solution lead to perturbative state mixing with nearby electronic states of different dipole moment. (v) For some molecules, the direction of the dipole moment changes upon electronic excitation what causes additional sources of errors.

The current scheme of thermochromic shifts with solvent polarity functions, that contain experimentally determined cavity volumes at each temperature, eliminates some of the above errors. Fig. 3 clearly shows that the cavity volume (and therefore also the cavity radius, assuming a spherical cavity) is a linear function of the temperature. Correct application of the equations describing the thermochromic shifts, therefore demands for the consideration of the temperature dependence of the cavity volume.

Other sources of error have been minimized in the current study by choosing the molecule carefully. The direction of the dipole moment in anisole is only slightly altered upon electronic excitation. Furthermore, the energetically following excited singlet state $\left(S_{2}\right)$ is $9200 \mathrm{~cm}^{-1}$ apart [55], minimizing the problem of perturbative state mixing. In subsequent studies, we will systematically vary the molecules according to these limitations in order to allow for more exact dipole moment determinations of excited state in the condensed phase.

\section{Conflicts of interest}

There are no conflicts to declare.

\section{Acknowledgements}

Financial support of the Deutsche Forschungsgemeinschaft via grant SCHM1043 12-3 is gratefully acknowledged. Computational support and infrastructure was provided by the "Center for Information and Media Technology" (ZIM) at the Heinrich-Heine-University Düsseldorf. We furthermore thank the Regional Computing Center of the University of Cologne (RRZK) for providing computing time on the DFG-funded High Performance Computing (HPC) system CHEOPS as well as support.

\section{References}

[1] P. Debye, Phys. Z. 13 (1912) 97.

[2] D.E. Williams, J. Comput. Chem. 9 (1988) 745-763.

[3] Baron, J. Phys. Chem. 89 (1985) 4873-4875.

[4] P. Suppan, N. Ghoneim, Solvatochromism, Royal Society of Chemistry, Cambridge, 1997.

[5] T. Förster, Ann. Phys. 437 (1948) 55-75.

[6] J. Lakowicz, Principles of Fluorescence Spectroscopy, 2nd ed., Plenum, New York, USA, 1999.

[7] H. van Amerongen, L. Valkunas, R. van Grondelle, Photosynthetic Excitons, World Scientific Publishing, Singapore, 2000.
[8] D.E. Freeman, W. Klemperer, J. Chem. Phys. 45 (1966) 52-57.

[9] J.R. Lombardi, D. Campbell, W. Klemperer, J. Chem. Phys. 46 (1967) 3482-3486.

[10] T.M. Korter, D.R. Borst, C.J. Butler, D.W. Pratt, J. Am. Chem. Soc. 123 (2001) 96-99.

[11] J.A. Reese, T.V. Nguyen, T.M. Korter, D.W. Pratt, J. Am. Chem. Soc. 126 (2004) 11387-11392.

[12] T. Nguyen, D. Pratt, J. Chem. Phys. 124 (1-6) (2006) 054317.

[13] P. Suppan, J. Photochem. Photobiol. A 50 (1990) 293.

[14] T. Abe, I. Iweibo, Bull. Chem. Soc. Jpn. 58 (1984) 3415-3422.

[15] W. Liptay, E. Lim (Ed.), EBST Dipole Moments and Polarizabilities of Molecules in Excited Electronic States, vol. I, Academic Press, New York, 1974, pp. 129-229 (Chapter 4).

[16] W. Liptay, Angew. Chem. 8 (1969) 177-188.

[17] E. Lippert, Z. Naturforsch. 10A (1955) 541.

[18] N. Mataga, Y. Kaifu, M. Koizumi, Bull. Chem. Soc. Jpn. 29 (1956) 465

[19] L. Onsager, J. Am. Chem. Soc. 58 (1936) 1486-1493.

[20] F.W. Fowler, A.R. Katritzky, R.J.D. Rutherford, J. Chem. Soc. B (1971) 460-469.

[21] R.W. Taft, J.-L.M. Abboud, M.J. Kamlet, M.H. Abraham, J. Solut. Chem. 14 (1985) $153-186$.

[22] T. Abe, Bull. Chem. Soc. Jpn. 64 (1991) 3224-3228.

[23] E.G. Demissie, E.T. Mengesha, G.W. Woyessa, J. Photochem. Photobiol. A 337 (2016) 184-191.

[24] A. Kawski, B. Kuklinski, P. Bojarski, Chem. Phys. Lett. 415 (2005) 251-255.

[25] C. Eisenhardt, G. Pietraperzia, M. Becucci, Phys. Chem. Chem. Phys. 3 (2001) 1407.

[26] J.W. Ribblett, W.E. Sinclair, D.R. Borst, J.T. Yi, D.W. Pratt, J. Phys. Chem. A 110 (2006) 1478-1483.

[27] M. Pasquini, N. Schiccheri, M. Becucci, G. Pietraperzia, J. Mol. Struct. 924 (2009) $457-460$.

[28] O. Desyatnyk, L. Pszczolkowski, S. Thorwirth, T.M. Krygowski, Z. Kisiel, Phys Chem. Chem. Phys. 7 (2005) 1708-1715.

[29] R. Ahlrichs, M. Bär, M. Häser, H. Horn, C. Kölmel, Chem. Phys. Lett. 162 (1989) $165-169$.

[30] J.T.H. Dunning, J. Chem. Phys. 90 (1989) 1007-1023.

[31] C. Hättig, F. Weigend, J. Chem. Phys. 113 (2000) 5154-5161.

[32] C. Hättig, A. Köhn, J. Chem. Phys. 117 (2002) 6939-6951.

[33] C. Hättig, J. Chem. Phys. 118 (2002) 7751-7761.

[34] A. Klamt, G. Schüürmann, J. Chem. Soc. Perkin Trans. 2 (1993) 799-805.

[35] W.L. Meerts, M. Schmitt, G. Groenenboom, Can. J. Chem. 82 (2004) 804-819.

[36] W.L. Meerts, M. Schmitt, Phys. Scripta 73 (2005) C47-C52.

[37] W.L. Meerts, M. Schmitt, Int. Rev. Phys. Chem. 25 (2006) 353-406.

[38] M. Schmitt, W.L. Meerts, Handbook of High Resolution Spectroscopy, John Wiley and Sons, 2011.

[39] A. Ostermeier, A. Gawelcyk, N. Hansen, Parallel Problem Solving from Nature, PPSN III, Springer, Berlin/Heidelberg, 1994.

[40] N. Hansen, A. Ostermeier, Evolutionary Computation 9 (2001) 159-195.

[41] J. Wilke, M. Wilke, W.L. Meerts, M. Schmitt, J. Chem. Phys. 144 (2016) 0442011-044201-10.

[42] E.M. Rae, J. Phys. Chem. 61 (1957) 562-572.

[43] L. Bilot, A. Kawski, Z. Naturforsch. 17A (1962) 621-627.

[44] I. Gryczyński, A. Kawski, Z. Naturforsch. 30A (1975) 287-291.

[45] S. Gerstenkorn, P. Luc, Atlas du spectre d'absorption de la molécule d'iode 14800$20000 \mathrm{~cm}^{-1}$, CNRS, Paris, 1986.

[46] M. Schmitt, Habilitation, Heinrich-Heine-Universität, Math. Nat. Fakultät, Düsseldorf, 2000.

[47] M. Schmitt, J. Küpper, D. Spangenberg, A. Westphal, Chem. Phys. 254 (2000) 349-361.

[48] M.M. Lindic, M. Zajonz, M.-L. Hebestreit, M. Schneider, W.L. Meerts, M. Schmitt, Data in brief (2018) (submitted for publication).

[49] D.B. Farmer, A. Holt, S. Walker, J. Chem. Phys. 44 (1966) 4116-4120.

[50] J.S. Muenter, V.W. Laurie, J. Chem. Phys. 35 (1966) 855-858.

[51] A. Kawski, Z. Naturforsch. 57A (2002) 255-262.

[52] A. Kawski, P. Bojarski, Spectrochim. Acta A 82 (2011) 527-528.

[53] J.R. Lombardi, J. Phys. Chem. A 102 (1998) 2817-2823.

[54] J.R. Lombardi, J. Phys. Chem. A 103 (1999) 6335-6338.

[55] R. Omidyan, H. Rezaei, Phys. Chem. Chem. Phys. 16 (2014) 11679-11689. 